\title{
Numerical and experimental investigations of stress wave propagation in utility poles under soil influence
}

\author{
N. Yan, J. Li, U. Dackermann \& B. Samali \\ Centre for Built Infrastructure Research, Faculty of Engineering and IT, University of Technology, Sydney, Australia
}

\begin{abstract}
Over the past decades, different types of non-destructive testing (NDT) methods have been developed for the condition assessment of timber structures. Stress wave methods, which are based on stress wave propagation theory, are considered to be simple and cost-effective tools for identifying damage as well as embedment length of timber poles/piles. However, due to the lack of understanding on wave propagation in such structures, especially under the influence of surroundings such as soil, these methods encounter difficulties in producing reliable results. In this paper, an investigation was carried out on the wave propagation behaviour in utility timber poles with consideration of soil effects. The commercial finite element (FE) analysis software ANSYS was used to simulate stress wave propagation in a timber utility pole under the influence of soil. In order to verify the numerical findings, laboratory testing was also conducted in conjuction with the numerical modelling.
\end{abstract}

\section{BACKGROUND}

Utility poles made of timber are traditionally used all over the world as they are relatively low cost and environmentally friendly. Especially in Australia, utility timber poles represent a significant part of the country's infrastructure for power distribution and communication networks. There are nearly 7 million timber poles in the current network in Australia, and among them, 5 million poles are used for power and communication supply (Nguyen et al., 2004). \$40-\$50 million are spent annually on maintenance and asset management to prevent utility lines from failure. The lack of reliable tools for assessing the condition of in-situ poles has seriously jeopardized maintenance and asset management. For example, in the Eastern States of Australia, about 300,000 electricity poles are replaced every year. However, up to $80 \%$ of them are still in a very good serviceable condition, causing a large waste of natural resources and money (Nguyen et al., 2004).

To address the needs of the utility pole asset management industry, a cost-effective and reliable NDT method needs to be developed that is able to identify and separate healthy from unhealthy poles. In the last 20 years, various NDT techniques have been developed to evaluate quality and conditions of timber structures. Among those methods/techniques, visual inspection and stress wave methods remain the most widely used techniques in practice (Tanasoiu et al., 2002). Visual inspection is undoubtedly one of the oldest methods used in practice but suffers a major drawback on reliability and accuracy of its results since the method relies on the experience of the operator and accessibility of the structure. Stress wave methods, on the other hand, can offer simple and cost-effective tools for identifying the in-service condition as well as the embedment length of timber poles/piles. However, due to lack of understanding of stress wave propagation in pole structures, especially with the effect of soil embedment coupled with unknown pole conditions below ground line (such as deterioration, moisture etc.), the results rely heavily on experience and interpretations from individual operators. Another issue influencing the testing accuracy is related to complexity of the timber material with anisotropy, non-homogeneity and many uncertainties on material properties. As a matter of fact, the material properties of timber can be affected heavily by environmental factors such as temperature and moisture changes. As a result, current stress wave NDT methods often fail to produce reliable results, which are vital for the utility pole management industry.

In order to address this pressing and critical issue, an industry supported research project started in 2011 that aims at developing accurate and reliable nondestructive assessment tools for timber poles based on stress wave techniques. One of the major components in this research is numerical investigations of stress wave propagation in timber poles considering effects of soil embedment and in-situ timber conditions. The commercial finite element analysis software ANSYS is the main tool used in this study. This paper presents some initial findings of the research which includes: i) development of a FE model for timber utility pole, ii) development of contact elements to simulate effects 
of surrounding soil to the stress wave propagation in timber pole, iii) investigation of the behaviour of stress wave propagation in timber poles, and iv) verification of the numerical results with experimental results.

\section{NDT FOR UTILITY TIMBER POLE INTEGRITY ASSESSMENT}

Stress wave methods including sonic and ultrasonic techniques are popular NDTs used in practice. Among various stress wave NDTs, surface wave NDTs, such as Sonic Echo (SE), Impulse Response (IR) and Bending Wave method, are known to be simple and costeffective but unreliable. In this paper, a study on the stress wave propagation in a FE model of a timber pole is extended for the application of a surface wave NDT, i.e. Sonic Echo, as verification of the FE model. A corresponding experimental testing is conducted and results are used for FE model verification.

The SE method is often used in pile/pole integrity testing by measuring the echoes (reflections) from either pole/pile foundation bottoms or defects of longitudinal (compressional) waves, which are generated by an impact hammer. The basic theory for this method is that propagating stress wave patterns may be changed after reflecting back when they encounter a boundary of different medium (such as the bottom of a structure or defects/damage) according to the impedance of the medium. The testing is performed by impacting the top of the pile/pole to generate longitudinal waves, which travel down to the bottom of the pile or pole. The wave will reflect back from boundary of a medium with different impedance including changes in stiffness, cross-sectional area and density. For example, a crack has lower impedance in comparison with the material of a timber pile, while much stiffer soil or bedrock layer has higher impedance (Larry et al., 1998). The reflected longitudinal wave is measured by a series of sensors. In this research, the experimental testing in the laboratory was conducted on a $5 \mathrm{~m}$ utility timber pole with $270 \mathrm{~mm}$ for the top diameter and $300 \mathrm{~mm}$ for the bottom diameter (see Fig. 1). The pole is embedded in soil by $1 \mathrm{~m}$ and eight PCB accelerometers with equal spacing are located along the surface of the pole in longitudinal direction. An impact load of $2.3 \mathrm{kN}$ is applied on the top centre of the pole by a modal impact hammer.

The estimation of the embedment length of a pole by $\mathrm{SE}$ method uses the measured echo time interval, according to the following formula:

$L_{t}=\frac{\beta \times V \times T}{2}$

where $L_{t}$ is the length between the location of the sensor and the bottom of the pole or location of a defect; $V$ is the velocity of the longitudinal wave; $T$ is the time difference between the first arrival event and the first reflection event recorded by the sensor. $\beta$ is a velocity adjustment factor and normally set to 0.9 .

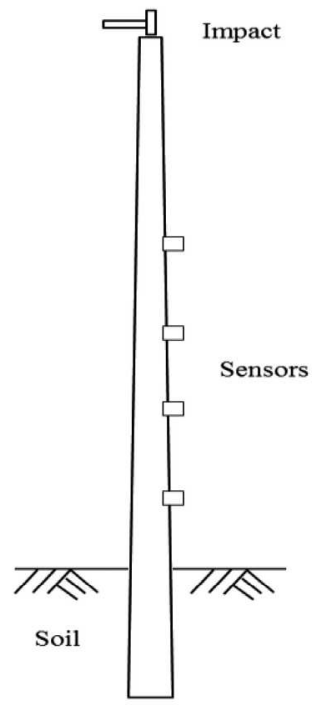

Figure 1. Sonic Fcho testing

As the frequency domain counterpart of the SE method, the IR method transfers the measured time history data into the frequency domain by Fast Fourier Transform (FFT). The length of the pole can be estimated by:

$L_{t}=\frac{\beta \times V}{2 \times F}$

where $F$ is the measured frequency interval between two consecutive resonant frequency peaks of the pole, and it is the inverse of $T$.

However, the velocity of the stress wave is affected by the moisture content in the wood. A typical timber pole in service contains a dry zone (above the ground), a saturated zone (below the ground), and a transient zone (an increasing amount of moisture). Therefore, the formula to estimate the length using measured data can be modified (Pandey and Anthony, 2005):

$L_{t}=L_{d}+L_{w}=L_{d}+\left(\frac{\beta \times V_{w}}{2 \times F}\right)-\frac{V_{w}}{V_{d}} \times L_{d}$

where $L_{\mathrm{d}}$ is the length of pole in the dry zone, $L_{\mathrm{w}}$ is the length of pole in the wet zone, $V_{\mathrm{d}}$ is the wave velocity of dry wood, and $V_{\mathrm{w}}$ is the wave velocity of the wet wood.

\section{DEVELOPMENT OF A FINITE ELEMENT} MODEL FOR TIMBER UTILITY POLES

Timber is a complex natural material with anisotropic and inhomogeneous characteristics. Its properties present a wide range of variability with time, loading rate, temperature and moisture content (Tabiei and $\mathrm{Wu}, 2000)$. The mechanical behaviour of timber 


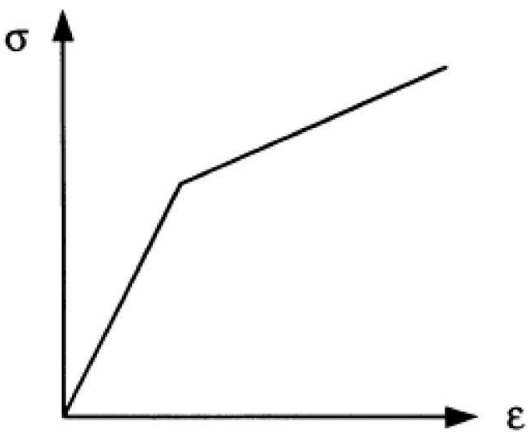

Figure 2. Stress-strain curve used to simulate the material behaviour of timber (Junior and Molina, 2010).

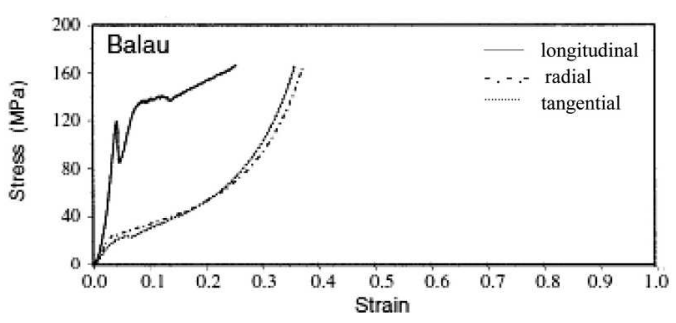

Figure 3. Stress/strain relationship for Balau loaded in compression up to a strain level of 1.0 (Ellis and Steiner, 2002).

is complicated and exhibits high nonlinearity. However, for applications involving small deformation, for the purpose of numerical modelling, timber is often simplified to be a linear-elastic and isotropic material with mean material properties (Tabiei and $\mathrm{Wu}, 2000$ ). Under large deformation scenarios, this simplification will not be valid and the model will not be able to represent the real behaviour of timber (Li, 2007). For analysing the failure and fatigue behaviour of timber structures, such as timber composite beams, it is well recognised that it is necessity to model timber as an orthotropic material (Serrano, 2000) with elastic-plastic behaviour (bilinear curve) based on Hill's criterion (Junior and Molina, 2010) (see Fig. 2).

In this research, the test specimens are hardwood timber poles made from Spotted Gum. The density ranges from $950 \mathrm{~kg} / \mathrm{m}^{3}$ (dry wood) to $1150 \mathrm{~kg} / \mathrm{m}^{3}$ (green wood), and the elastic modulus is from $18 \mathrm{GPa}$ (green wood) to $23 \mathrm{GPa}$ (dry wood) (Bootle, 1983).

A typical stress-strain behaviour of hardwood can be illustrated by hard wood species named Balau with the density of $979 \mathrm{~kg} / \mathrm{m}^{3}$ (see Fig. 3).

It is well known that at a low strain level (for example, less than 0.03), the timber exhibits linear elastic behaviour (Ellis and Steiner, 2002). In this project, by numerical analysis, it was shown that an impact hammer induced stress and strain is below 0.001 through the entire pole. Therefore, it is reasonable to use linear elastic model to simulate timber materials.

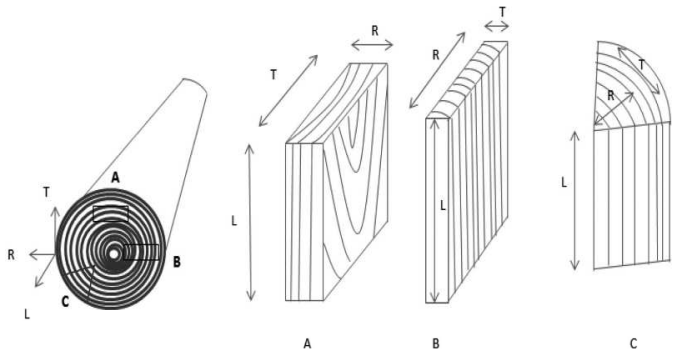

Figure 4. Three dimensional nature of timber fibre.

In the establishment of the constitutive model for timber material, based on the characteristics of timber, several models have been used by researchers to simulate the behaviour in timber poles:

1. idealised isotropic and homogeneous continuum model; orthotropic and homogeneous continuum model;

2. transversely isotropic (the identical properties in tangential and radial directions) and homogeneous continuum model (Murray, 2007).

In the linear-elastic region, the orthotropic elasticity material constitutive equation can be expressed by equation (4). In the equation, $\varepsilon$ is elastic strain vector, $\gamma$ is the shear strain vector, $\gamma$ is stress vector, $E$ is the elastic modulus, $G$ is the shear modulus and $\nu$ is the Poison's ratio. The subscripts $L, R$ and $T$ refer to the directions along the grain (longitudinal direction), across the grain (radius direction), and tangential to the grain (tangential direction), respectively (see Fig. 4).

$$
\left[\begin{array}{c}
\varepsilon_{L L} \\
\varepsilon_{R R} \\
\varepsilon_{T T} \\
\gamma_{L R} \\
\gamma_{L T} \\
\gamma_{R T}
\end{array}\right]=\left[\begin{array}{ccccccc}
\frac{1}{E_{L}} & -\frac{v_{R L}}{E_{R}} & -\frac{v_{T L}}{E_{T}} & 0 & 0 & 0 \\
-\frac{v_{L R}}{E_{L}} \frac{1}{E_{R}} & -\frac{v_{T R}}{E_{T}} & 0 & 0 & 0 \\
-\frac{v_{L T}}{E_{L}} & -\frac{v_{R T}}{E_{R}} \frac{1}{E_{T}} & 0 & 0 & 0 \\
0 & 0 & 0 & \frac{1}{G_{L R}} & 0 & 0 \\
0 & 0 & 0 & 0 & \frac{1}{G_{L T}} & 0 \\
0 & 0 & 0 & 0 & 0 & \frac{1}{G_{R T}}
\end{array}\right] \times\left[\begin{array}{c}
\sigma_{L L} \\
\sigma_{R R} \\
\sigma_{T T} \\
\tau_{L R} \\
\tau_{L T} \\
\tau_{R T}
\end{array}\right](4)
$$

The matrix of Eq. 4 can be simplified to:

$\{\varepsilon\}=[C]_{\text {orth }}\{\sigma\}$

where $\{\varepsilon\}$ is the strain tensor, $\{\sigma\}$ is the stress tensor and $[\mathrm{c}]_{\text {orth }}$ is the orthotropic elastic matrix $(6 \times 6)$. As in orthotropic material,

$\frac{v_{\mathrm{ij}}}{\mathrm{E}_{\mathrm{i}}}=\frac{v_{\mathrm{ji}}}{\mathrm{E}_{\mathrm{j}}}, \mathrm{i} \neq \mathrm{j} \mathrm{i}, \mathrm{j}=\mathrm{L}, \mathrm{R}, \mathrm{T}$

Hence, $[\mathrm{c}]_{\text {orth }}$ can be written as a symmetric matrix, and nine parameters are needed to define the timber material properties. 
In this paper, as a preliminary investigation of stress wave behaviour in a timber pole, timber is modelled as linear elastic isotropic material. For the next stage of this research, both linear elastic orthotropic and transverse isotropic models will be considered for timber constitutive modelling.

\section{DEVELOPMENT OF CONTACT ELEMENTS}

To model a timber utility pole in service, soil embedment needs to be properly modelled. The selection of a suitable soil model is usually related to the type of loading and the objectives of the investigation. Dynamic load testing of piles or poles is often divided into two types: high strain dynamic load testing and low strain dynamic load testing. High strain dynamic load testing is typically used to evaluate the carrying capacity and integrity of driven piles (Thasnanipan et al., 2000). Low strain dynamic load testing, on the other hand, is used to inspect integrity and embedment length to assess the condition of piles (Kirsch and Plabmann, 2002). In this research, low strain integrity testing, namely SE testing, is conducted to evaluate the integrity and embedment length of utility timber poles and thus, linear elastic behaviour will be considered for soil simulation.

To accurately and realistically model the interaction between timber utility pole and surrounding soil for stress wave propagation is a challenging task. In this paper, two interaction simulation methods, i.e. coupling method and contact method, are investigated.

For applications with small deflections or rotations and two components bonded together without friction, the problem can be simplified to a linear analysis, where the coupling method can be used to simulate interfacial action between the pole and the soil (ANSYS, 2011). In the coupling method, the pole and soil are fully connected, which means at interface the degree of freedom of pole and soil is exactly the same and both act as a whole unit when stress waves move through.

Normally, the contact method in ANSYS is used to simulate highly nonlinear and complicated interfacial behaviour between two components. There are different types of contact methods available in ANSYS. In this paper, the "node to node" contact method is more suitable for capturing interfacial behaviour between the pole and soil.

"Node to node" contact method is often used where the relative sliding between interfacial components is small or the deformation is negligible, which is the case in this project. In this research, an investigation is conducted comparing the coupling method against the contact method, with the "node to node" contact method being selected to simulate the interaction between soil and timber pole. The contact behaviour is considered as no separation and sliding is permitted to simulate the potential situation in experimental testing. In terms of the computation algorithms based on the understanding from experimental testing, the
Table 1. Parameters for modelling.

\begin{tabular}{llll}
\hline Material & $\begin{array}{l}\text { Density } \\
\left(\mathrm{kg} / \mathrm{m}^{3}\right)\end{array}$ & $\begin{array}{l}\text { Elastic Modulus } \\
\left(\mathrm{N} / \mathrm{m}^{2}\right)\end{array}$ & $\begin{array}{l}\text { Poison's } \\
\text { Ratio }\end{array}$ \\
\hline Timber Pole & 950 & $2.3 \mathrm{E} 10$ & 0.3 \\
Soil & 1520 & $1.0 \mathrm{E} 8$ & 0.3 \\
\hline
\end{tabular}

Lagrange Multiplier on normal plane and penalty on tangent plane are considered, as this algorithm is suitable for a very small amount of slip for a sticking contact condition. Since no reference was found for setting the parameters to simulate interaction between timber and soil, all the parameters for defining the contact behaviour are tentatively set up and are subjected to further study at the next stage of this research.

\section{INVESTIGATION OF STRESS WAVE PROPAGATION BEHAVIOUR IN TIMBER POLES}

In this paper, the timber material and soil are considered as isotropic and linear elastic for FE modelling. The parameters used are listed in Table 1.

The overall length of timber pole is $5 \mathrm{~m}$ with the top diameter being $27 \mathrm{~cm}$ and the bottom diameter being $30 \mathrm{~cm}$. The surrounding soil is $1.2 \mathrm{~m}$ in length, $1.2 \mathrm{~m}$ in width and $3 \mathrm{~m}$ in depth, which is exactly the same as in the experimental case. The timber pole is embedded $1 \mathrm{~m}$ into the soil. The 3-D model is created with solid 185 element in ANSYS. Both coupling and contact methods are applied to simulate the interaction behaviour between the soil and the timber pole. A hammer impact is imparted at the centre on the top of the pole and transient analysis is conducted to obtain time history stress wave data. The hammer impact induces stress waves propagating through the pole from the top to the bottom and reflecting back. The sensors (represented by nodes) capture the incident wave (the first peak) as well as the reflected wave (the second peak).

\subsection{Contact method}

Figure 5 shows the numerical results based on contact method, captured from the sensors at different locations from the bottom of the pole. To get a clearer picture of the first two peaks, Figure 6 provides a zoomed-in view.

As the incident wave propagates through the pole, each sensor captures the wave in sequence from the top to the bottom. As a result, the wave velocity can be calculated based on the sensor locations and time delays. From Eq. (7), the average wave velocity (group velocity) can be obtained, which is $4,872 \mathrm{~m} / \mathrm{s}$.

$V=\frac{D_{2}-D_{1}}{T_{2}-T_{1}}$ 


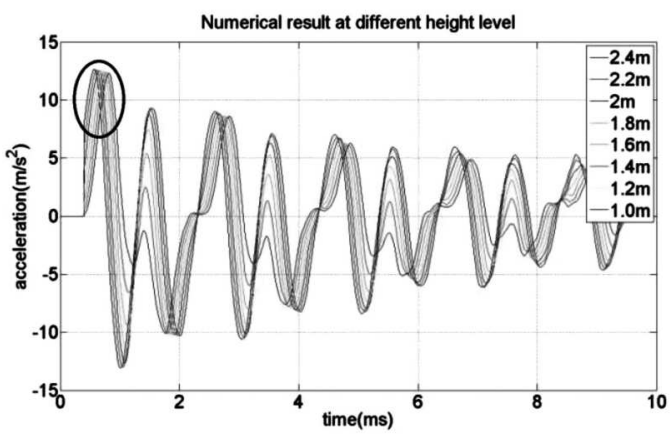

Figure 5. Numerical results using the contact method.

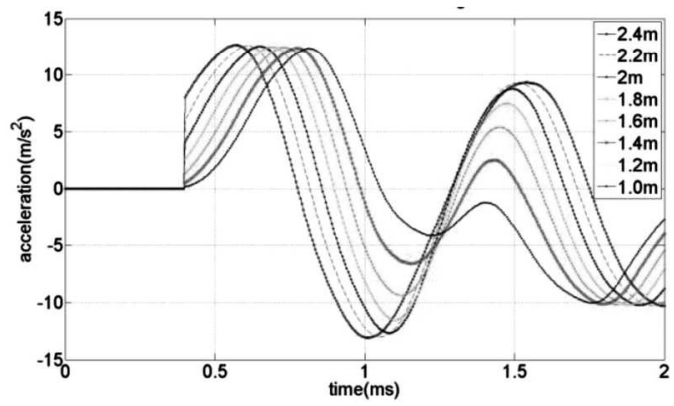

Figure 6. Zoomed-in view of the first two peaks.

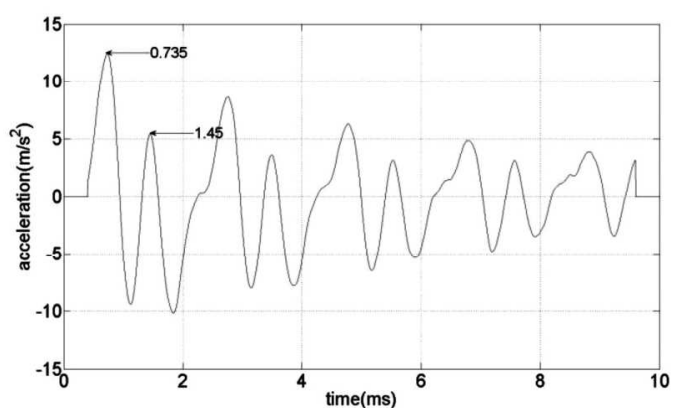

Figure 7. Numerical data from sensor at $1.6 \mathrm{~m}$ (contact method).

where $D$ is sensor location and $T$ the time record of the first peak. According to Eq. (1), the embedment length of the pole can also be calculated.

As an example, Figure 7 displays the numerical results captured from the sensor at $1.6 \mathrm{~m}$. Here, the first peak is reached at $0.735 \mathrm{~ms}$ and the reflected peak is reached at $1.45 \mathrm{~ms}$. From Eq. (1), the length between the sensor and bottom of the pole is obtained as $1.57 \mathrm{~m}$, which gives a $2 \%$ error compared to the true length.

\subsection{Coupling method}

Figure 8 shows the numerical results using the coupling method. Again, stress waves are captured by the sensors at various sensor locations (nodes).

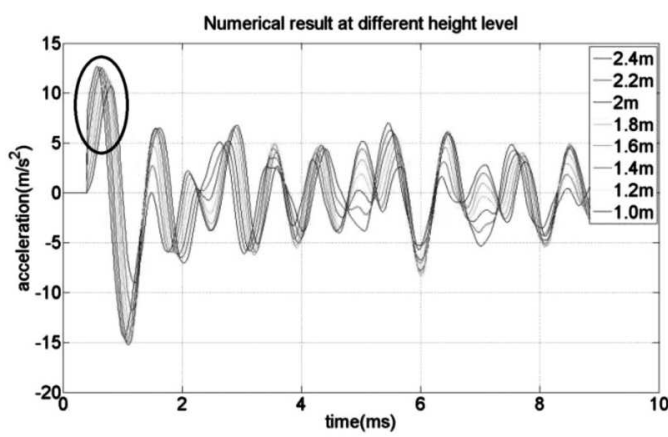

Figure 8. Numerical results using the coupling method.

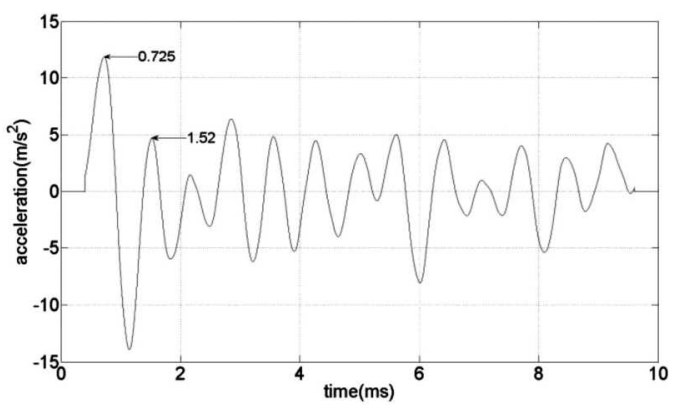

Figure 9. Numerical data from sensor at $1.6 \mathrm{~m}$ (coupling method).

The same calculation yields a velocity of $5,200 \mathrm{~m} / \mathrm{s}$, which is much higher than the one obtained by the contact method. The distance between the sensor at $1.6 \mathrm{~m}$ and the bottom of the pole can again be calculated based on the incident wave peak and the reflected wave peak

Using Eq. (1), the distance between the sensor and the bottom pole is obtained as $1.86 \mathrm{~m}$, which presents a $16.3 \%$ error in relation to the true length.

\section{VERIFIFACATION OF THE NUMERICAL RESULTS WITH EXPERIMENTAL RESULTS}

To verify the numerical modelling, a comparison between experimental and numerical results was conducted. In Figures 10 and 11, the thick curve displays filtered experimental data captured by the sensor at $1.6 \mathrm{~m}$ and the thin curve displays the numerical results at the same location.

\subsection{Verification of the model created using the contact method}

The time records of the first two peaks are critical for the SE and other echo based NDTs in order to estimate the underground embedment length of the pole. Using the time records of the first two peaks from experimental results, the length between the sensor and the bottom of pole is $1.55 \mathrm{~m}$, which is quite close to 


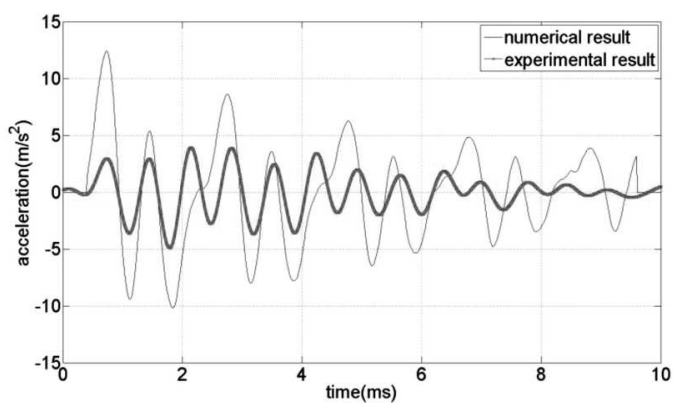

Figure 10. Comparison of experimental and numerical results using the contact method.

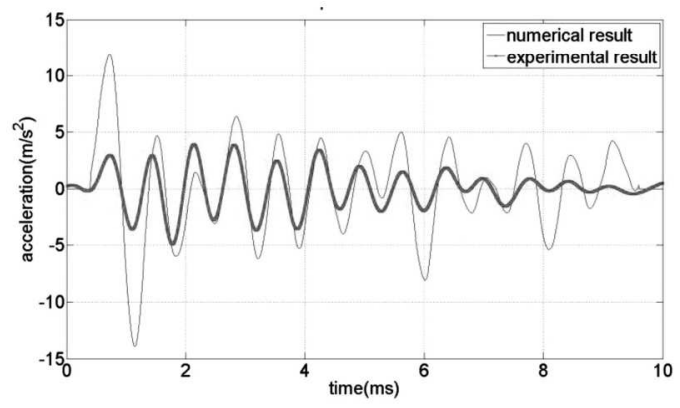

Figure 11. Comparison of experimental and numerical results using the coupling method.

the true length with a $3.1 \%$ error. From Figure 10, it is observed that the locations of the first two peaks for numerical and experimental results are correlated. However, other peaks and wave patterns after $2 \mathrm{~ms}$ are not correlated well.

\subsection{Verification of the model created using the coupling method}

From Figure 11, it is observed that using the coupling method, the overall trend of the stress wave of the numerical simulation matches well with the experimental results. However, the time record of the second peak of the numerical result does not match with the experimental results. As a result, it produces a lager error in the estimation of the embedment length.

\section{CONCLUSION}

This paper presents an investigation on numerical modelling of stress wave propagation in utility poles under soil influence. Two interfacial methods, namely, the coupling method and the contact method, were proposed to simulate the interfacial behaviour between a timber pole and its surrounding soil for stress wave propagation. Experimental testing was also conducted to verify the numerical findings. The results showed that the coupling method can produce better overall wave propagation patterns in simulating the stress wave behaviour in the timber pole. On the other hand, the contact method can produce accurately the first two peaks of the wave pattern, which is crucial in determination of the underground embedment length, but experiences a problem in simulating the overall behaviour of the stress wave. Further investigations on these two methods will be carried out in the next stage of the project.

\section{REFERENCES}

ANSYS, Inc. 2011. User's manual for ANSYS' 13.0. Canonsburg, Pennsylvania, U.S. patent application.

Bootle, K. R. 1983. Wood in Australia, Australia, McGraw-Hill Australia Pty Ltd.

Ellis, S. \& Steiner, P. 2002. The Behaviour of Five Wood Species in Compression. International Association of Wood Anatomists Journal, 23(2):201-211.

Junior, C. C. \& Molina, J. C. 2010. Numerical Modelling Strategy for Analysing the Behaviour of Shear Connectors in Wood-Concrete Composite Systems. World Conference on Timber Engineering. Italy.

Kirsch, F. \& Plabmann, B. Dynamic Methods in Pile Testing: Developments in Measurement and Analysis. Deep Foundations 2002: An International Perspective on Theory, Design, Construction, and Performance:868-882 Orlando, Florida, U.S.

Larry, M., Olson, D., Jalinoos, F. \& Aouad, M. F. 1998. Determination of Unknown Subsurface Bridge Foundation. Federal Lands Highway Program Administration.

Li, M. B. 2007. Thesis of Modelling and Simulation of Wood Mechanical Properties Based on Finite Element Theory. $\mathrm{PhD}$, Northeast Forest Univeristy, China.

Murray, Y. D. 2007. Manual for LS-DYNA Wood Material Model-143. U.S.: Research, Development, and Technology Turner-Fairbank Highway Research Center.

Nguyen, M., Foliente, G. \& Wang, X. 2004. State of the Practice and Challenges in Non-destructive Evaluation of Utility Poles in Service. Key Engineering MaterialsAdvances in non-destructive evaluation: 270-273.

Pandey, A. K. \& Anthony, R. W. 2005. Inspection and monitoring techniques for bridges and civil structures. England: The Institute of Materials, Minerals \& Mining.

Serrano, E. 2000.Thesis of Adhesive Joints in Timber Engineering-Modelling and Testing of Fracture Properties. PhD, I und University, Sweden.

Tanasoiu, V., Miclea, C. \& Tanasoiu, C. 2002. Non-destructive Testing Techniques and Piezoelectric Ultra-Sonics Transducers for Wood and Built in Wooden Structures. Journal of Optoelectronics and Advanced Materials, 4.

Thasnanipan, N., Maung, A. W., Navaneethan, T. \& Aye, Z. Z 2000. Non-destructive integrity testing on piles founded in Bangkok subsoil. Application of Stress-Wave Theory to Piles: 171-177. Brazil: Balkema Ratterdam, Brazil.

Tabiei, A. \& Wu, J. 2000. Three-dimensional nonlinear orthotropic Finite element material model for wood. Composite Structures, 50(2000), 143-149. 